\title{
DEVELOPING SPRING-ROLL DIELECTRIC ELASTOMER ACTUATOR SYSTEM BASED ON NEW OPTIMAL DESIGN PARAMETERS
}

\author{
Medhat H. A. Awadalla*and Besada A. Anees** \\ *Electronics and Communication Dept., Faculty of Engineering, Helwan \\ University, Cairo,Egypt E-mail:awadalla_medhat@yahoo.co.uk \\ **Industrial Training Council., Cairo, Egypt. \\ E-mail: bessada adib@yahoo.com
}

(Received June 30, 2011 Accepted July 20, 2011)

To develop a well designed practical spring-roll dielectric elastomer actuator system, we have to optimize the design parameters of spring-roll dielectric elastomer and develop a voltage supply that is able to pump enough controlled charges and provide a wide range of controlled voltages to the actuator. In this paper, switched based multistage charge pump driven by a controlled low voltage switching power supply and a voltage driver is proposed as a wide range output voltage supply.

Spring-roll dielectric elastomer optimal design parameters according to [1] are $\eta_{1}{ }^{p}=5, \eta_{2}{ }^{p}=2, \alpha=10$. It is proven that $\eta_{2}{ }^{p}$ has a subtle effect on both actuation $\lambda_{1}$ and dimensionless axial force $p / \mu L_{2} L_{3}$ however it has a great effect on the applied voltage required activating the actuator. Furthermore, when $\lambda_{2}{ }^{p}$ increases, the applied voltage decreases and the problems of high voltage are avoided. Therefore the optimal design parameters modified to $\lambda_{1}^{p}=5, \lambda_{2}^{p}=5, \alpha=10$ instead of $\lambda_{1}^{p}=5, \lambda_{2}^{p}=2$, $\alpha=10$.

In this paper, based on the equations of state, new approaches for designing a spring-roll dielectric elastomer actuator have been developed. The key issue of the actuation range depends upon weather the actuator has a fixed load or variable multi-loads.

The achieved results based on the proposed approaches show that the applied voltage has been reduced and the design of the actuator is getting simpler.

KEY WORDS: Spring roll dielectric elastomer actuator, modes of failure, optimal design parameters, switching power supply, charge pump.

\section{INTRODUCTION}

Dielectric elastomer actuators have been intensely studied in the recent decade. When the actuator is subjected to an applied voltage and an applied axial force, the axial elongation couples the electrical and mechanical actions. The construction of a springroll dielectric elastomer actuator was indicated in [2-5].

Providing dielectric elastomer actuators with a level of pre-stretch can improve properties such as breakdown strength, actuation strain and efficiency [6]. The 
parameters of design include the prestretches of the elastomer and the stiffness of the spring.

Equations of state of the dielectric elastomer actuator were derived from its Helmholtz free energy. Equations of state namely are;

$$
\begin{aligned}
& \frac{P}{\mu L_{2} L_{3}}=\left(\lambda_{1}-\lambda_{1}^{-3}\left(\lambda_{2}^{p}\right)^{-2}\right)-\frac{1}{\lambda_{1}^{3}\left(\lambda_{2}^{p}\right)^{2}}\left(\frac{Q}{\sqrt{\mu \varepsilon} L_{1} L_{2}}\right)^{2}+\alpha\left(\lambda_{1}-\lambda_{1}^{p}\right) \\
& \frac{\Phi}{L_{3}} \sqrt{\frac{\varepsilon}{\mu}}=\frac{1}{\left(\lambda_{1} \lambda_{2}^{p}\right)^{2}}\left(\frac{Q}{\sqrt{\mu \varepsilon} L_{1} L_{2}}\right)
\end{aligned}
$$

Where;

$P:$ is the axial force which the actuator is loaded with.

$\mu$ : is the shear modulus of the dielectric elastomer material.

$L_{1}, L_{2}$, and $L_{3}$ : are the actuator membrane length, width, and thickness.

$\lambda_{1}$ : is the elongation or actuation.

$\lambda_{1}^{p}, \lambda_{2}^{p}$ and $\lambda_{3}^{p}$ : are pre-stretches in actuator membrane length, width, and thickness.

$\alpha:$ is the spring stiffness.

$Q$ : is the charge accumulated on one of the electrodes of the dielectric elastomer actuator.

$\varepsilon:$ is the dielectric of the elastomer material.

$\Phi$ : is the voltage applied to the electrodes of the dielectric elastomer actuator.

The range of operation of an actuator is limited by various modes of failure. Each mode of failure restricts the state of the actuator to a region on the plane of the generalized coordinates. The common region that averts all modes of failure constitutes the set of allowable states [7-9]. The modes of failure namely are;

Electromechanical instability (EMI) that is represented mathematically by;

$\frac{Q}{\sqrt{\mu \varepsilon} L_{1} L_{2}}=\sqrt{(1+\alpha) \lambda_{1}^{4}\left(\lambda_{2}^{p}\right)^{2}+3}$

Electrical breakdown $(\mathrm{EB})$ that is represented mathematically by;

$\frac{Q}{\sqrt{\mu \varepsilon} L_{1} L_{2}}=\lambda_{1} \lambda_{2}^{p} E_{C} \sqrt{\frac{\varepsilon}{\mu}}$

where $E_{C}$ is the critical electric field at which breakdown occurs.

Loss of tension $\left(S_{1}\right)$ due to the stress in the axial direction [10] that is represented mathematically by;

$$
\frac{Q}{\sqrt{\mu \varepsilon} L_{1} L_{2}}=\sqrt{\lambda_{1}^{4}\left(\lambda_{2}^{p}\right)^{2}-1}
$$

Loss of tension $\left(S_{2}\right)$ due to the stress in the circumferential direction that is represented mathematically by; 
$\frac{Q}{\sqrt{\mu \varepsilon} L_{1} L_{2}}=\sqrt{\lambda_{1}^{2}\left(\lambda_{2}^{p}\right)^{4}-1}$

Tensile rupture of the elastomer occurs when the values of $\lambda_{1}^{p}, \lambda_{2}^{p}$, or $\lambda_{1}$ are exceeding the critical value $\lambda_{C}>5$. Therefore the values of the above mentioned stretches should not exceed 5.

Compressive limit of the spring which occurs at $\lambda_{1}=\lambda_{1}^{p} / 4$.

The continuation of the paper is as follows: A new concept of actuation range is introduced in section 2. Optimal design parameters are prescribed in section 3, design approaches and samples of actuator design are presented in section 4. Actuator system voltage supply is proposed in section 5. Section 6 gives the conclusion and future work

This paper is an extension to our work in [1] where enhancements to optimal design parameters, actuation range concept, and spring-roll dielectric elastomer actuator design procedures have been presented and a new charge pump design is introduced as a controllable voltage supply.

\section{ACTUATION RANGE}

The actuation range is an important issue because the applied voltage may take different values within the actuation range.

In this paper the concept of actuation range depends upon whether the actuator has a fixed load actuator or variable multi-load actuator. The fixed load actuator is the actuator subject to a fixed axial force (i.e., a dead weight). In this case the actuation range is very small value starting at the state of zero charge, and ending at the state where the line of a failure mode intersects the axial force line. Fig. 1 shows the actuation range for a fixed load actuator. The actuation range extends from $\lambda_{1}=4.4$ to $\lambda_{1}=4.515 \quad$ (the two highlighted black points) when $\lambda_{1}^{p}=5, \lambda_{2}^{p}=5, \alpha=10, P / \mu L_{2} L_{3}=-1$, and $E_{C}=10^{8} v / m$.

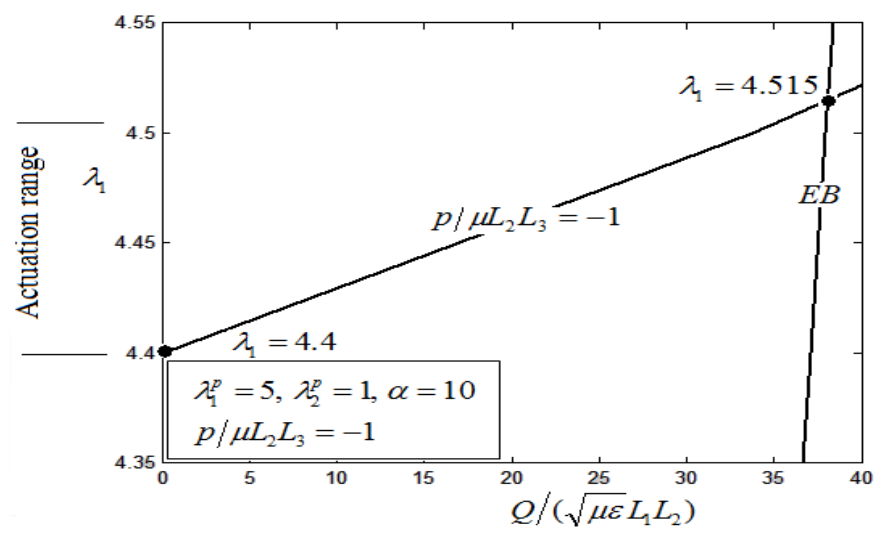

Fig. 1: The actuation range for a fixed load actuator when $\lambda_{1}^{p}=5, \lambda_{2}^{p}=5, \alpha=10, P / \mu L_{2} L_{3}=-1$, and $E_{C}=10^{8} v / \mathrm{m}$. 
The load of the exchangeable multi-load actuator can be replaced by another unequal load and this load in turn can be replaced by another unequal one and so on. The actuation range of the exchangeable multi-load actuator starts at the state of zero charge of heaviest load and ends at the state where the line of a failure mode intersects the line of the lightest load. Fig. 2 shows the actuation range of the exchangeable multi-load actuator. The actuator given in Fig. 2 subjects to one of the following dimensionless loads; $P / \mu L_{2} L_{3}=-1, \quad P / \mu L_{2} L_{3}=-10, \quad P / \mu L_{2} L_{3}=-20$, or $P / \mu L_{2} L_{3}=-30$. The actuation range in this case starts at $\lambda_{1}=1.95$ and ends at $\lambda_{1}=4.52$ (the two highlighted black points).

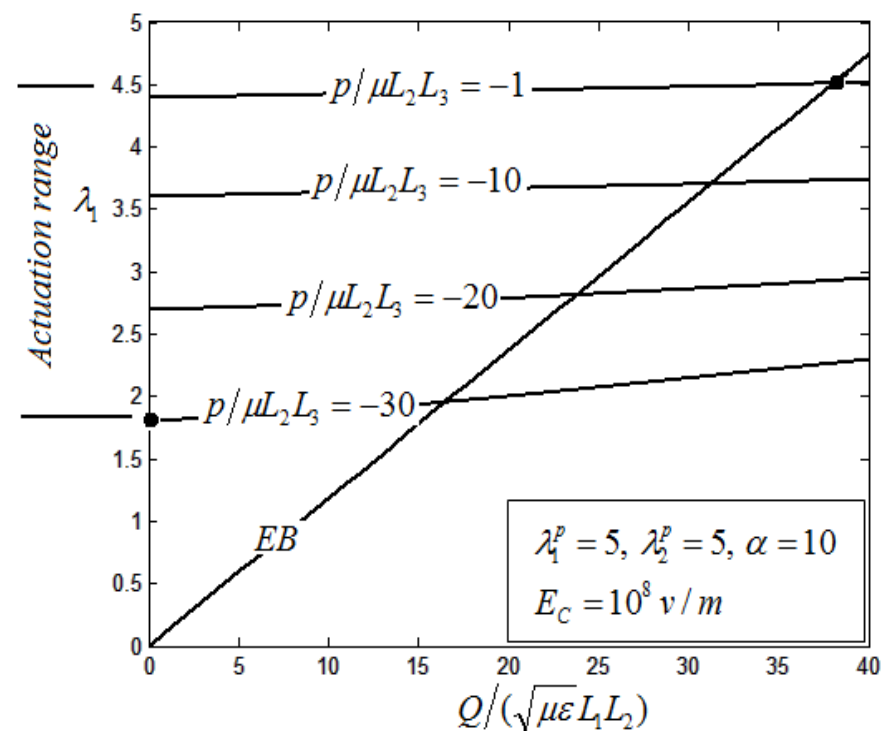

Fig. 2: The actuation range of the exchangeable multi-load actuator.

\section{SPRING-ROLL DIELECTRIC ELASTOMER ACTUATOR OPTIMAL DESIGN PARAMETERS}

Parameters of design are prescribed once the actuator is constructed therefore they must well studied and selected to be optimal.

To optimize an actuator, the actuator should have certain features such as higher actuation and the ability to burden heavier loads. To address this issue, we have to figure out the space of design parameters values that confirm these features.

Spring-roll dielectric elastomer optimal design parameters according to [1] are $\lambda_{1}^{p}=5, \lambda_{2}^{p}=2, \alpha=10$. These parameters of design are prescribed once the actuator is constructed.

In this paper we will address the effect of $\lambda_{2}^{p}$ on actuation $\lambda_{1}$, axial force $P / \mu L_{2} L_{3}$, and $\Phi /\left(L_{3} \sqrt{\mu / \varepsilon}\right)$ to prove that the value $\lambda_{2}^{p}=2$ that was considered as an optimal value should be changed to $\lambda_{2}^{p}=5$. 


\subsection{The Actuation $\lambda_{1}$ as a Function of $\lambda_{2}^{p}$}

The obtained results in simulation show that when $\lambda_{2}^{p}$ increases, the actuation $\lambda_{1}$ slightly decreases. There is no big difference between actuation at $\lambda_{2}^{p}=1$ and actuation at $\lambda_{2}^{p}=5$ even at higher values of the dimensionless charge. From fig. 3 , it is clear that $\lambda_{2}^{p}$ slightly affects $\lambda_{1}$.

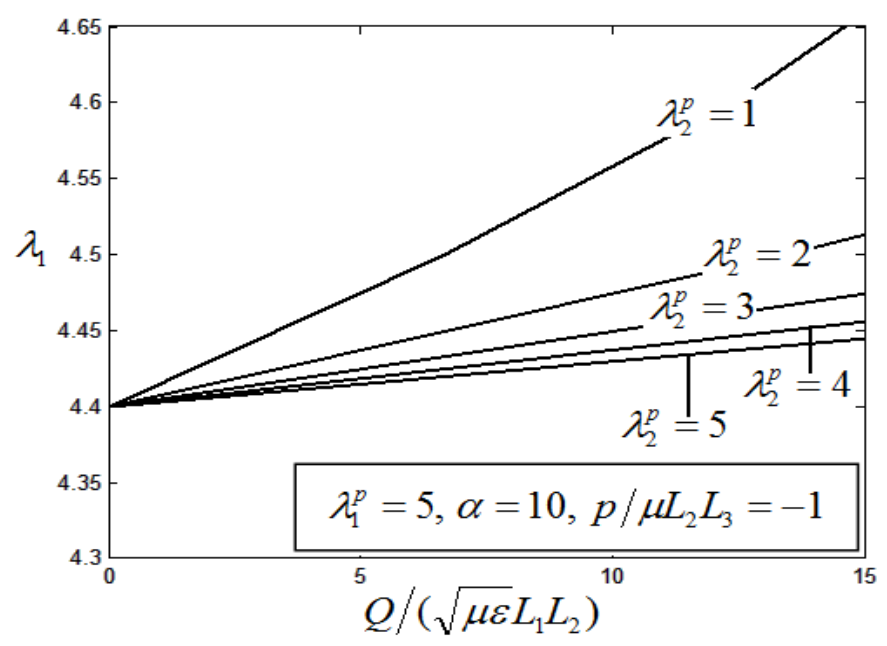

Fig. 3: The effect of $\lambda_{2}^{p}$ on the actuation $\lambda_{1}$ at $\lambda_{1}^{p}=5, \alpha=10$, and $P / \mu L_{2} L_{3}=-1$

\subsection{The Dimensionless Force (load) $P / \mu L_{2} L_{3}$ as a Function of $\lambda_{2}^{p}$}

The dimensionless axial force $P / \mu L_{2} L_{3}$ is a nonlinear function of $\lambda_{2}^{p}$. $\lambda_{2}^{p}$ Slightly affects the value (modulus) of the axial force. Fig. 4 shows the effect of $\lambda_{2}^{p}$ on the axial force.

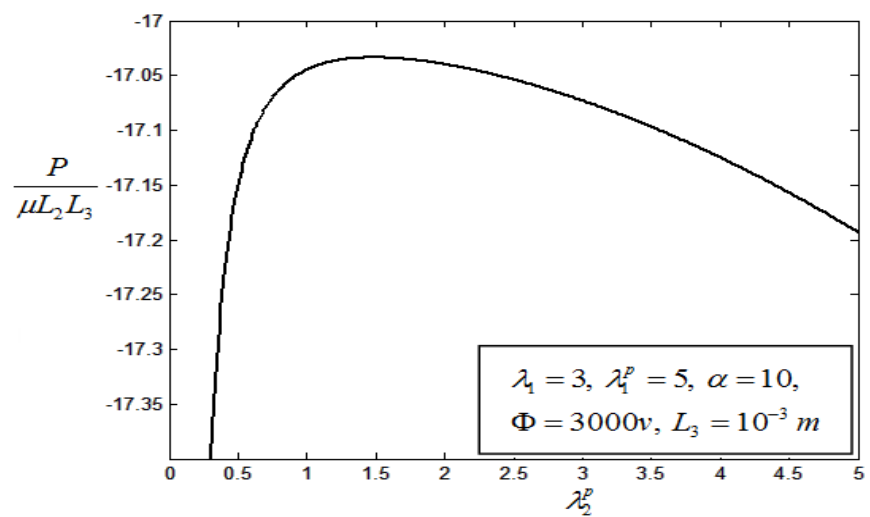

Fig. 4: The effect of $\lambda_{2}^{p}$ on the axial force $P / \mu L_{2} L_{3}$ at $\lambda_{1}=3, \lambda_{1}^{p}=5, \alpha=10$,

$$
L_{3}=10^{-3} \text {, and } \Phi=3000 v
$$




\subsection{The Effect of $\lambda_{2}^{p}$ on the Applied Voltage $\Phi$}

The work in paper [1] did not take the applied voltage into consideration as a measure or standard for selecting optimal design parameters. Because if the applied voltage is addressed, the optimal value of $\lambda_{2}^{p}$ might be changed.

$\lambda_{2}^{p}$ slightly affects both actuation $\lambda_{1}$ and dimensionless axial load $p / \mu L_{2} L_{3}$ but it has a great effect on the applied voltage $\Phi$. When $\lambda_{2}^{p}$ increase, the applied voltage decreases. Let us take the applied voltage at a critical state $\Phi_{C}$ where one failure mode sets in as an example for the relation between the applied voltage and $\lambda_{2}^{p}$. When $\lambda_{1}^{p}=5, \lambda_{2}^{p}=1, \alpha=10, p / \mu L_{2} L_{3}=0$, and $L_{3}=1 \mathrm{~mm}$, the critical dimensionless applied voltage is 0.215 and the critical applied voltage is $12756 v$, as shown in Fig. 5.

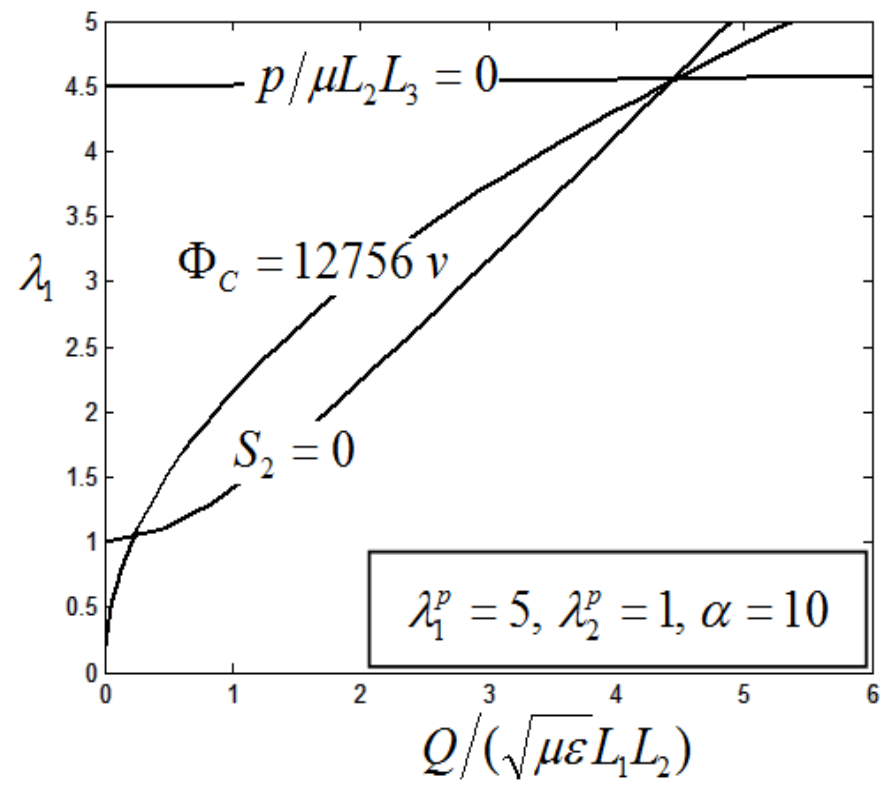

Fig. 5: The critical applied voltage at

$$
\lambda_{1}^{p}=5, \lambda_{2}^{p}=1, \alpha=10, p / \mu L_{2} L_{3}=0 \text {, and } L_{3}=1 \mathrm{~mm} \text {. }
$$

When $\lambda_{1}^{p}=5, \lambda_{2}^{p}=2, \alpha=10, p / \mu L_{2} L_{3}=0$, and $L_{3}=1 \mathrm{~mm}$, the critical dimensionless applied voltage is 0.182 and the critical applied voltage is $10798 v$, look at Fig. 6.

When $\lambda_{1}^{p}=5, \lambda_{2}^{p}=3, \alpha=10, p / \mu L_{2} L_{3}=0$, and $L_{3}=1 \mathrm{~mm}$, the critical dimensionless applied voltage is 0.122 and the critical applied voltage is $7238 v$, as shown in Fig. 7. 


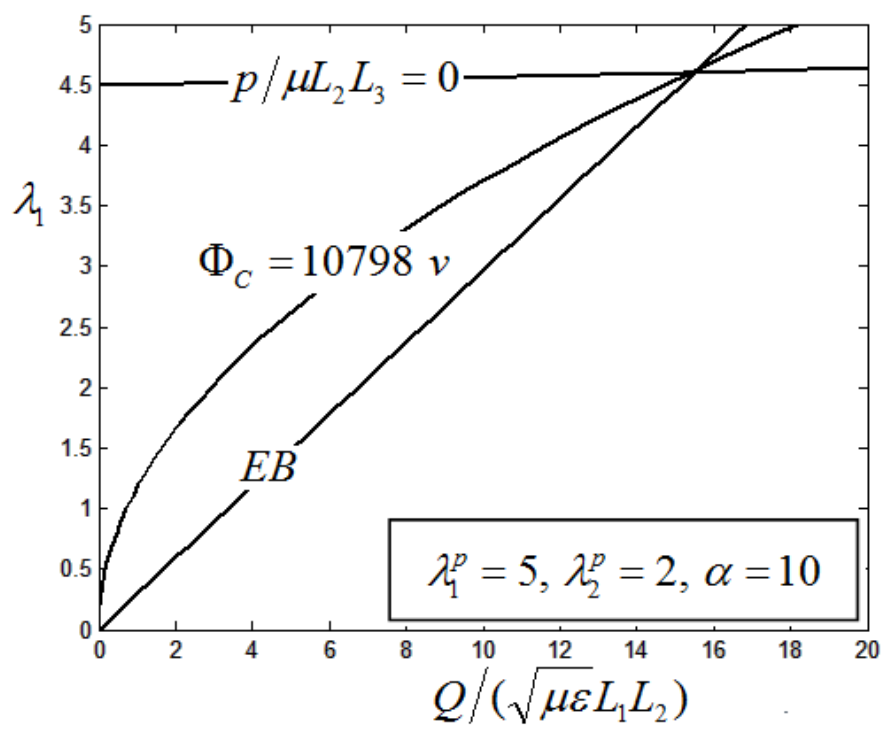

Fig. 6: The critical applied voltage at

$\lambda_{1}^{p}=5, \lambda_{2}^{p}=2, \alpha=10, p / \mu L_{2} L_{3}=0$, and $L_{3}=1 \mathrm{~mm}$.

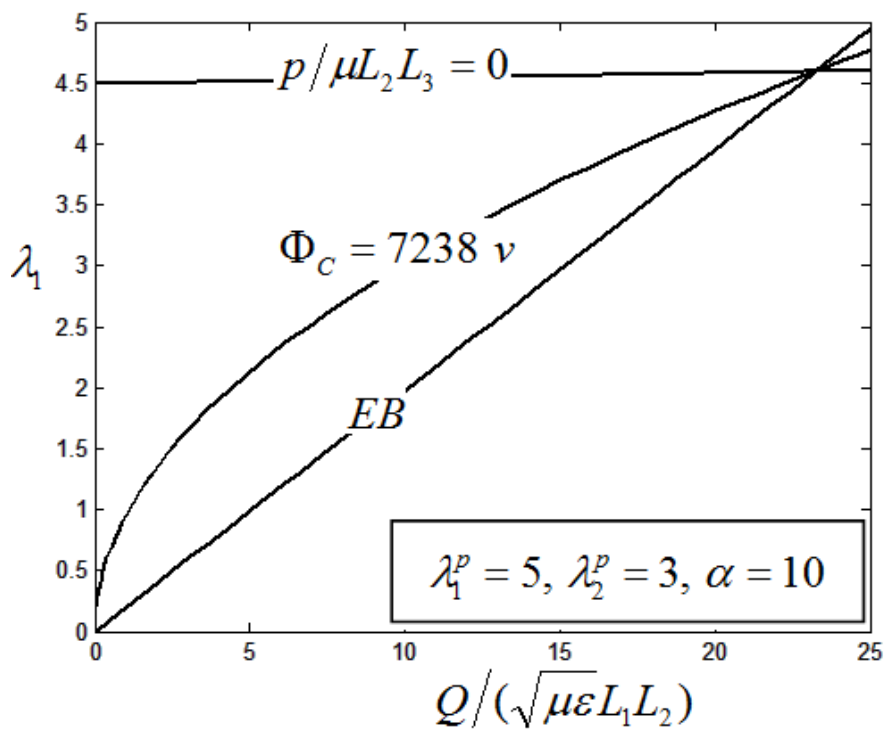

Fig. 7: The critical applied voltage at

$\lambda_{1}^{p}=5, \lambda_{2}^{p}=3, \alpha=10, p / \mu L_{2} L_{3}=0$, and $L_{3}=1 \mathrm{~mm}$.

When $\lambda_{1}^{p}=5, \lambda_{2}^{p}=4, \alpha=10, p / \mu L_{2} L_{3}=0$, and $L_{3}=1 \mathrm{~mm}$, the critical dimensionless applied voltage is 0.092 and the critical applied voltage is $5458 v$, look at Fig. 8 . 


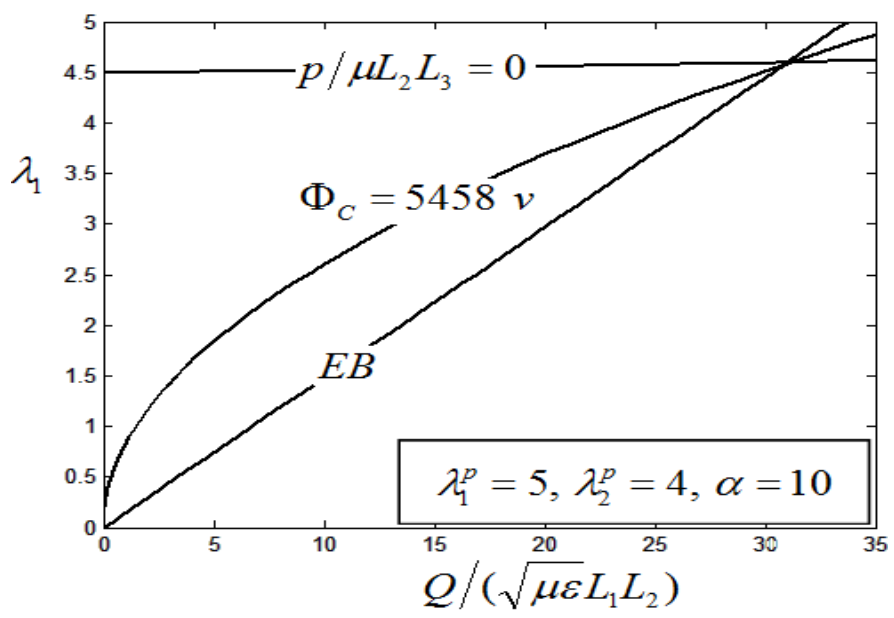

Fig. 8: The critical applied voltage at

$\lambda_{1}^{p}=5, \lambda_{2}^{p}=4, \alpha=10, p / \mu L_{2} L_{3}=0$, and $L_{3}=1 \mathrm{~mm}$.

When $\lambda_{1}^{p}=5, \lambda_{2}^{p}=5, \alpha=10, p / \mu L_{2} L_{3}=0$, and $L_{3}=1 \mathrm{~mm}$, the critical dimensionless applied voltage is 0.073 and the critical applied voltage is $4331 v$, look at Fig. 9.

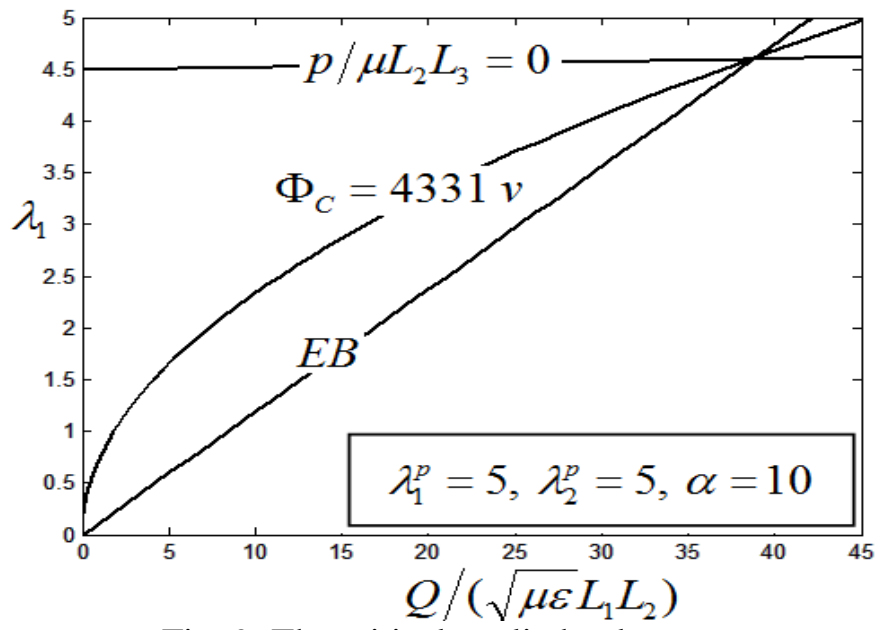

Fig. 9: The critical applied voltage at

$\lambda_{1}^{p}=5, \lambda_{2}^{p}=5, \alpha=10, p / \mu L_{2} L_{3}=0$, and $L_{3}=1 \mathrm{~mm}$.

The voltage at a critical state $\Phi_{C}$ decreases from $12756 \mathrm{v}$ at $\lambda_{2}^{p}=1$ to $4331 \mathrm{v}$ at $\lambda_{2}^{p}=5$. In general, the applied voltage decreases as $\lambda_{2}^{p}$ increases and high voltage problems can be avoided therefore the optimal design parameters should be changed from;

$$
\lambda_{1}^{p}=5, \lambda_{2}^{p}=2, \alpha=10 \quad \text { to } \quad \lambda_{1}^{p}=5, \lambda_{2}^{p}=5, \alpha=10
$$




\section{SPRING-ROLL DIELECTRIC ELASTOMER DESIGN PROCEDURES}

Spring-roll dielectric elastomer actuator is designed according to equations of state (eq. 5 , and eq. 6). The first step of designing the elastomer actuator is to be aware of the constant values of equations of state;

$\mu$ : The shear modulus of the dielectric elastomer (VHB 4910 material) equals $10^{5}$ Pascal [11].

$\varepsilon_{r}$ : The relative dielectric constant equals 3.21 for VHB 4910 material, at a frequency of $1 \mathrm{KHz}$.

$\varepsilon_{o}$ : The permittivity equals $8.85 \times 10^{-12} \mathrm{Farad} /$ meter.

$E_{C}$ : The electric breakdown of VHB 4910 material equals $10^{8} \mathrm{v} / \mathrm{m}$.

$\lambda_{1}^{p}, \lambda_{2}^{p}, \alpha$ : The design parameters are dealt as constant values; $\lambda_{1}^{p}=5, \lambda_{2}^{p}=5, \alpha=10$.

$L_{3}$ : The thickness of the dielectric elastomer (VHB 4910 material), 3M company produces it in a thickness of $1 \mathrm{~mm}$ therefore it is dealt as a constant value.

\subsection{Spring-Roll Dielectric Elastomer Specifications Prescribed by the Customer}

The customer has to specify three specifications before the actuator is constructed; axial force/forces in Newtons, length of the actuator at relax $L_{1 \text { relax }}$ in meters, and actuator maximum required length $L_{1 \max }$ in meters. Dimensionless axial force/forces should not be positive (tensile force/forces) and should not situate in the region of modes of failure. If this happens, the customer is reviewed to modify his request.

4.2 Determining the Maximum Actuation (stretch) $\lambda_{1 \max }$

$L_{1 \text { relax }}=\lambda_{1}^{p} L_{1}$

$L_{1 \text { max }}=\lambda_{1 \text { max }} L_{1 \text { relax }}$

$\lambda_{1 \max }=L_{1 \max } / L_{1 \text { relax }}$

\subsection{Determining the Range of the Applied Voltage}

The applied voltage may take infinite different values along the actuation range starting at $\Phi=0$ and ending at $\Phi=\Phi_{C} . E=\Phi / \lambda_{3} L_{3}$ where $E$ is the electric field across the elastomer membrane. When $\Phi=\Phi_{C}, E=E_{C}$, where $E_{C}$ is the electric breakdown field. Then $E_{C}=\Phi_{C} / \lambda_{3} L_{3}$. Since the dielectric elastomer is incompressible material, $\lambda_{3}=1 /\left(\lambda_{2}^{p} \lambda_{1}\right)$.

$\Phi_{C}=\frac{10^{8} L_{3}}{\lambda_{2}^{p} \lambda_{1}}$ 
where;

$E_{C}=10^{8} v / m$ for VHB 4910 dielectric elastomer material.

$\lambda_{2}^{p}$ according to new derived optimal design parameters equals " 5 ".

3 M company produces VHB 4910 membrane in a thickness of $1 \mathrm{~mm}$. $\Phi_{C}$ can be determined.

\subsection{Determining $L_{2}$, the Width of the Elastomer Membrane}

Substituting $\lambda_{1 \max }$ and $\Phi_{C}$ in eq. 6 , dimensionless charge $Q /\left(\sqrt{\mu \varepsilon} L_{1} L_{2}\right)$ can be determined. Substituting the determined $Q /\left(\sqrt{\mu \varepsilon} L_{1} L_{2}\right)$ and $\lambda_{1 \max }$ in eq. $7, L_{2}$ can be determined.

\subsection{Determining $\mathrm{K}$, the Stiffness of the Spring}

$L_{1}=L_{1 \text { relax }} / \lambda_{1}^{p}, L_{2}$ has been determined in section 6.4. Substituting $L_{1}$ and $L_{2}$ in the following eq; $\alpha=K L_{1} / \mu L_{2} L_{3}, K$ can be determined.

\subsection{Determining n, Number of Turns of Actuator Membrane Around the Spring}

Approximately the number of turns of the actuator membrane can be determined by dividing the width of the actuator $L_{2}$ by the circumference of the spring.

$$
n=\frac{\lambda_{2}^{p} L_{2}}{2 \pi r}
$$

where; $r$ is radius of the used spring.

\subsection{Samples of Spring-Roll Dielectric Elastomer Design Results}

In appendix A we develop equations of state based on Mat-Lab program help design spring-roll dielectric elastomer actuators. The customer has to specify the relaxed length of the actuator $L_{1 \text { relax }}$, the maximum required length the actuator has to achieve $L_{1 \max }$, and axial load the actuator will treat with. Using the above mentioned software, we can design the following actuator specifications; the dimensions of the actuator membrane, the stiffness of the used spring, the maximum applied voltage, and maximum achieved actuation. Table 1 includes samples of design specifications where the dimensions $L_{1}, L_{2}, L_{3}, L_{1 \max }, L_{1 \text { relax }}$, and $r$ are measured in meters, $\Phi_{\max }$ or $\Phi_{\text {critical }}$ is measured in volts, stiffness of the spring $k$ is measured in newtons per meter, and the axial load $p$ is measured in newtons. 
Table 1: Samples of spring-roll dielectric elastomer design specifications

\begin{tabular}{|c|c|c|c|c|c|c|c|c|c|c|}
\hline$p$ & $L_{1 \text { relax }}$ & $L_{1 \text { max }}$ & $r$ & $L_{1}$ & $L_{2}$ & $L_{3}$ & $\Phi_{c}$ & $\lambda_{1 \max }$ & $k$ & $n$ \\
\hline-4 & 0.01 & 0.02 & 0.001 & 0.02 & 0.0014 & 0.001 & 10000 & 2 & 679.7 & 1.08 \\
\hline-8 & 0.02 & 0.05 & 0.001 & 0.004 & 0.0034 & 0.001 & 8000 & 2.5 & 846 & 2.7 \\
\hline-12 & 0.03 & 0.09 & 0.001 & 0.006 & 0.0067 & 0.001 & 6667 & 3 & 1114 & 5.3 \\
\hline-16 & 0.04 & 0.14 & 0.0025 & 0.008 & 0.013 & 0.001 & 5714 & 3.5 & 1624 & 4.14 \\
\hline-20 & 0.05 & 0.05 & 0.0025 & \multicolumn{7}{|c|}{ The actuator is overloaded } \\
\hline-24 & 0.06 & 0.07 & 0.0025 & \multicolumn{7}{|c|}{ The actuator is overloaded } \\
\hline+28 & 0.07 & 0.14 & 0.0025 & \multicolumn{7}{|c|}{ The load is a tensile forces } \\
\hline+32 & 0.08 & 0.184 & 0.0025 & \multicolumn{7}{|c|}{ The load is a tensile force } \\
\hline-36 & 0.09 & 0.225 & 0.005 & 0.0180 & 0.0152 & 0.001 & 8000 & 2.5 & 846 & 2.4 \\
\hline-40 & 0.1 & 0.29 & 0.005 & 0.02 & 0.021 & 0.001 & 6897 & 2.9 & 1048 & 3.3 \\
\hline-44 & 0.11 & 0.352 & 0.005 & 0.022 & 0.028 & 0.001 & 6250 & 3.2 & 1274 & 4.5 \\
\hline-48 & 0.12 & 0.42 & 0.005 & 0.024 & 0.039 & 0.001 & 5714 & 3.5 & 1624 & 6.2 \\
\hline-52 & 0.13 & 0.234 & 0.005 & 0.026 & 0.0164 & 0.001 & 11111 & 1.8 & 629 & 2.6 \\
\hline-56 & 0.14 & 0.294 & 0.005 & 0.028 & 0.0198 & 0.001 & 9524 & 2.1 & 708 & 3.2 \\
\hline-60 & 0.15 & 0.345 & 0.005 & 0.03 & 0.231 & 0.001 & 8696 & 2.3 & 771 & 3.7 \\
\hline-64 & 0.16 & 0.432 & 0.005 & 0.032 & 0.03 & 0.001 & 7407 & 2.7 & 937 & 4.8 \\
\hline-68 & 0.17 & 0.527 & 0.005 & 0.034 & 0.0404 & 0.001 & 6452 & 3.1 & 1189 & 6.4 \\
\hline-72 & 0.18 & 0.702 & 0.005 & 0.036 & 0.092 & 0.001 & 5128 & 3.9 & 2555 & 14.6 \\
\hline-76 & 0.19 & 0.76 & 0.01 & 0.038 & 0.1132 & 0.001 & 5000 & 4 & 2980 & 9 \\
\hline-80 & 0.2 & 0.82 & 0.01 & 0.04 & 0.143 & 0.001 & 4878 & 4.1 & 3576 & 11.4 \\
\hline-84 & 0.21 & 0.399 & 0.01 & 0.042 & 0.0275 & 0.001 & 10526 & 1.9 & 654 & 2.2 \\
\hline-88 & 0.22 & 0.484 & 0.01 & 0.044 & 0.0325 & 0.001 & 9090 & 2.2 & 738 & 2.6 \\
\hline-92 & 0.23 & 0.598 & 0.01 & 0.046 & 0.0409 & 0.001 & 7692 & 2.6 & 889 & 3.3 \\
\hline-96 & 0.24 & 0.576 & 0.01 & 0.048 & 0.0387 & 0.001 & 8333 & 2.4 & 807 & 3.1 \\
\hline+100 & 0.25 & 0.975 & 0.01 & & & ne loa & a ten & force & & \\
\hline
\end{tabular}

\section{SPRING-ROLL DIELECTRIC ELASTOMER ACTUATOR VOLTAGE SUPPLY}

In spring-roll dielectric elastomer actuator system to achieve a specific actuation $\lambda_{1}$, a specific voltage should be applied to the electrodes of the actuator and specific charges should flow to them, therefore a voltage supply whose output ranges from several volts 
up to $15000 v$ is required. This voltage supply should be adjusted automatically to any voltage between 0 and $15000 \mathrm{v}$ whatever the value of the required voltage is.

A charge pump driven by a low voltage switching power supply and a voltage drive is presented in this paper [12].

\subsection{Charge Pump}

Increased voltage levels are obtained in a charge pump as a result of transferring charges to a capacitive load and do not involve amplifiers or transformers. The charge pump is constructed by $n$ cascaded voltage doublers. Charge pump operates by switching on and off a large number of switches which charge and discharge a large number of capacitances, transferring energy to the output load. Switched-capacitor charge pumps have exponentially growing voltage gain as a function of the number of stages (voltage doublers) up to $2^{n}$ [13].

A switched-capacitor organization of a two phase DC-DC voltage doubler is shown in Fig. 10. It contains 2 clock controlled switches and 2 capacitors.

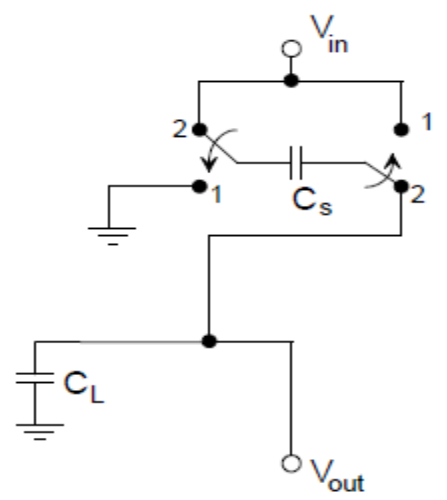

Fig. 10: The DC-DC TPVD voltage doubler

For a simple explanation of the voltage doubler operation, let us assume that the switches and capacitors are all ideal. That is, we assume that there is no leakage current in capacitors, switches dissipate no energy and the electric charge transferring is instantaneous. Fig. 11.a shows the equivalent circuit of the voltage doubler when the circuit is in the Kth iteration cycle and the clock is in phase 1. At this time instance, the load capacitor $C_{L}$ holds the previous voltage value.

$$
V_{\text {out }}^{[K]}=V_{\text {out }}^{[K-1]}
$$

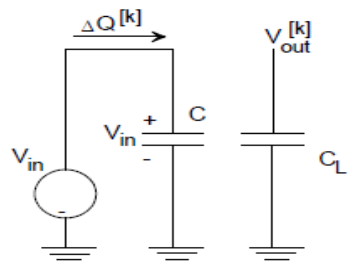

(a)

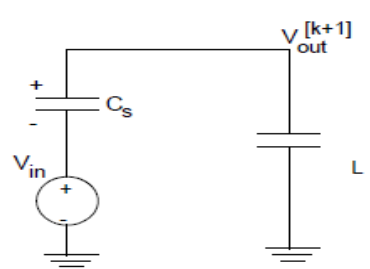

(b)

Fig. 11: The equivalent circuits in two clock phases. 
The voltage across the capacitor $C_{S}$ changes from $-\left(V_{\text {in }}-V_{\text {out }}^{[K-1]}\right)$ to $V_{\text {in }}$. The charge $\Delta Q^{[K]}$ transferred from the voltage source $V_{i n}$ to $C_{S}$ is obtained from $\Delta Q^{[K]}=C_{S}\left(V_{\text {in }}-\left(-\left(V_{\text {in }}-V_{\text {out }}^{[K-1]}\right)\right)\right)=C_{S}\left(2 V_{\text {in }}-V_{\text {out }}^{K}\right)$

Equation (15) implies that the voltage source $\mathrm{V}_{\text {in }}$ would stop transferring charge to $C_{S}$ if $V_{\text {out }}^{[K]}=2 V_{\text {in }}$.

Figure 11.b shows the equivalent circuit of the voltage doubler when the circuit is in the $(k+1)$ th iteration cycle and the clock is in phase 2 . According to the charge conservation law at the node connecting $C_{S}$ and $C_{S}$, and evaluating charges stored in capacitors as $Q=C V$, the relationship between voltages at $k$ th and $(k+1)$ th iteration can be expressed by

$V_{\text {in }} \times C_{S}+V_{\text {out }}^{[K]} \times C_{L}=\left(V_{\text {out }}^{[K+1]}-V_{\text {in }}\right) \times C_{S}+V_{\text {out }}^{[K+1]} \times C_{L}$

If we set $r=\frac{C_{S}}{C_{L}+C_{S}}$, to represent the capacitor ratio, then

$V_{\text {out }}^{[K+1]}=(1-r) V_{\text {out }}^{K}+2 r V_{\text {in }}$

where $0<r<1$. Thus, $V_{\text {out }}$ can be represented as a sequence of the iteration index $k$.

Figure 12 shows the voltage gains $A v$ as a function of the iteration index $k$, with different $r$. The smaller the $r$, the larger the ratio of the grounded capacitor $C_{L}$ to the switched capacitor $C s$. It is clear that the final (steady state) value of the voltage gain $A v$ is 2 independently of the capacitor ratio $r$. That is, the circuit in Fig. 11 works as a voltage doubler provided that the voltage source $V_{\text {in }}$ supplies enough charge to the charge pump. The larger $C_{L}$ (smaller $r$ ) requires more clock cycles (bigger $k$ ) to reach the desired output voltage. The value of $r$ does not influence the final voltage gain.

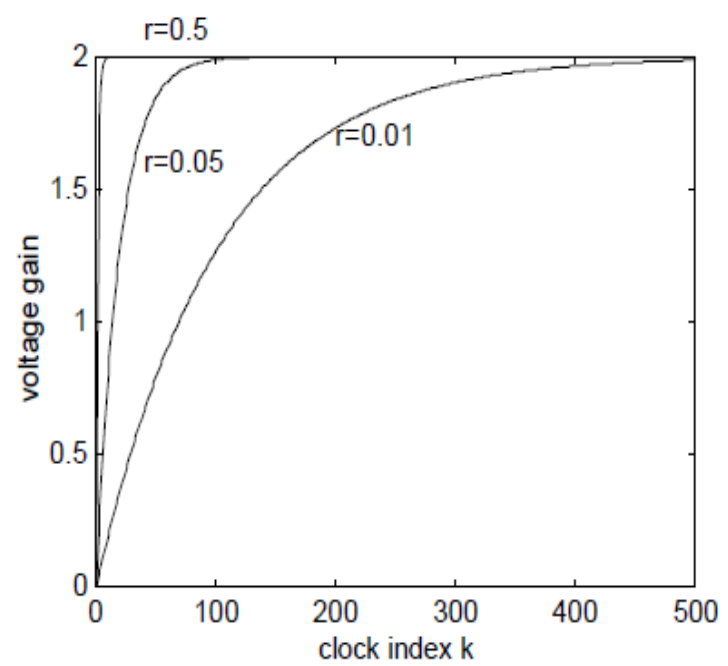

Fig. 12: The voltage gain as a function of the clock index $k$ 


\subsection{Proposed Actuator Voltage Supply}

A new organization of spring-roll dielectric elastomer actuator voltage supply is presented in this paper. Switched multistage charge pump driven by a controlled low voltage switching power supply and a voltage driver is used as a variable output high voltage supply. Coarse adjustment of the output voltage is automatically accomplished by connecting a specific number of the stages of the charge pump to the actuator. Fine adjustment of the output voltage is automatically accomplished by controlling the width of the pulse of the low voltage switching power supply. Fig. 13 shows the block diagram of the proposed voltage supply.

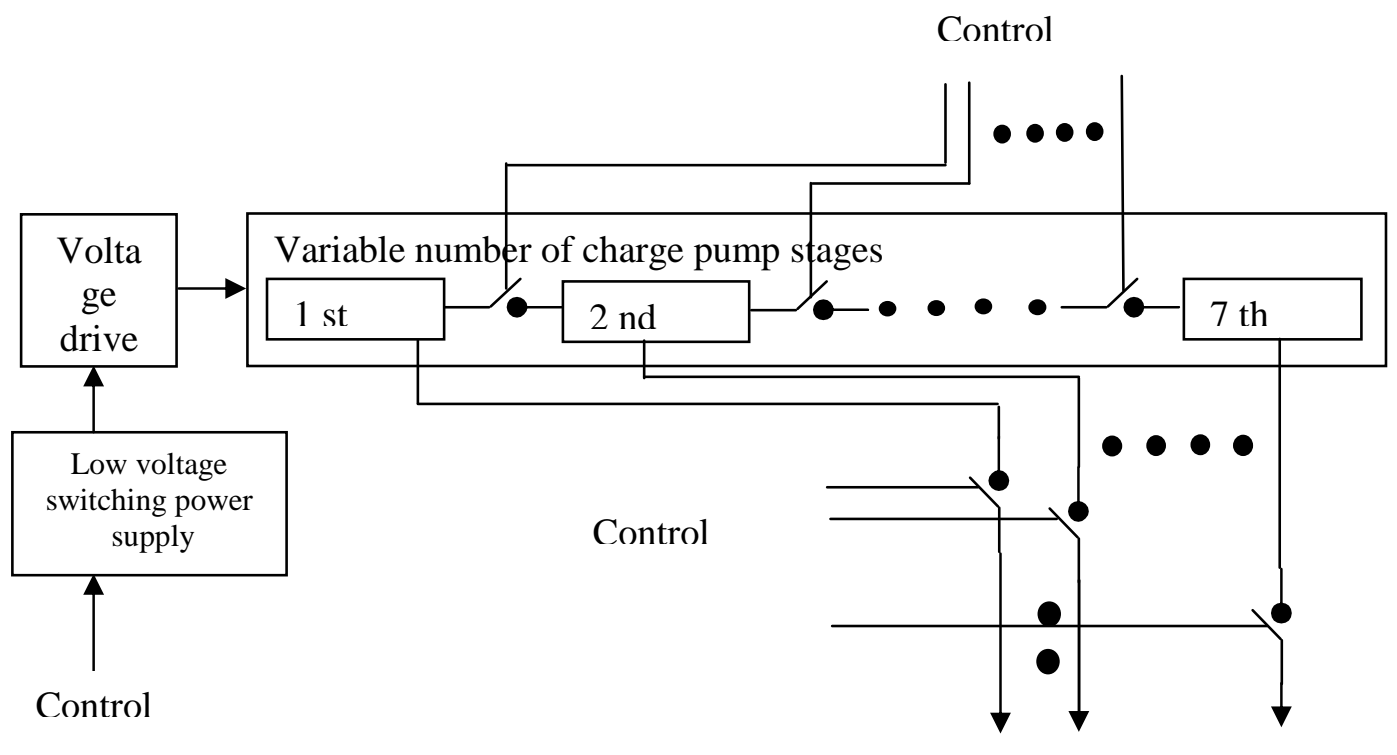

Fig. 13: Block diagram of the proposed voltage supply.

The voltage driver is designed to supply 120 volt at a $50 \%$ duty cycle in the low voltage switching power supply. The input and output voltages of each stage of the charge pump are indicated in table 2.

Table [2]: The input and output and output of each stage of the charge pump

\begin{tabular}{|c|c|c|}
\hline $\begin{array}{c}\text { Charge pump } \\
\text { stage number }\end{array}$ & $\begin{array}{c}\text { Input } \\
\text { voltage }\end{array}$ & $\begin{array}{c}\text { Output } \\
\text { voltage }\end{array}$ \\
\hline 1 & 120 & 240 \\
\hline 2 & 240 & 480 \\
\hline 3 & 480 & 960 \\
\hline 4 & 960 & 1920 \\
\hline 5 & 1920 & 3840 \\
\hline 6 & 3840 & 7680 \\
\hline 7 & 7680 & 15360 \\
\hline
\end{tabular}


At reset state all switches are switched off, duty cycle of low voltage switching power supply is $50 \%$ and the output of the voltage driver is 120 volt. Suppose that the actuator needs instantaneous 4000 volt to achieve a specific actuation, the control circuit connects stages umber 1,2,3,4, and 5 in series and the output is taken from the fifth stage (the automatic coarse adjustment), then the control circuit increases the duty cycle (pulse width) of the voltage switching power supply until the output of the fifth stage reaches 4000 volt (the automatic fine adjustment).

\section{CONCLUSION}

In this paper we search two problems, the first is to develop an optimal design parameters based spring-roll dielectric elastomer actuator, and the second is to develop a controlled wide range output high voltage supply. As for the first problem, we prove that $\lambda_{2}^{p}$ has slight effect on both actuation $\lambda_{1}$ and axial load, but has a high effect on the applied high voltage, it reduces the required applied high voltage down to $35 \%$ when raised to 5 . Therefore the optimal design parameters are changed to; $\lambda_{1}^{p}=5, \lambda_{2}^{p}=5, \alpha=10$.

In this paper we develop procedures of designing a spring-roll dielectric elastomer actuator, involve a software help designing the actuator [appendix 1], and mention samples of the results of designed spring-roll dielectric elastomer actuators.

In this paper the concept of actuation range depends upon whether the actuator is a fixed load actuator or an exchangeable multi-loads actuator.

As for the second problem a switched multistage charge pump driven by a controlled low voltage switching power supply and a voltage driver is proposed as a wide range output high voltage supply.

Solving the two problems a complete spring-roll dielectric elastomer actuator system is developed.

For future work, the instantaneous change of actuation of the spring-roll dielectric elastomer actuator is required. This actuation can be controlled by controlling both charges pumped to the electrodes of the actuator and the voltage applied to them. In a next paper we shall develop a sub control system to control charges pumped to electrodes of the actuator and another sub control system to control the applied voltage. Modes of failure of the actuator will be avoided using the proposed control system. Sensors of charges and sensors of high voltage will be used. Intelligent system will be proposed.

\section{REFERENCES}

[1] Medhat H. Ahmed and Besada A. Anees, "Parameter Optimization in Spring-Roll Dielectric Elastomer Actuator Design“,The Journal of Engineering Sciences, Faculty of Engineering, University of Assiut, Assiut, Egypt, 2011.

[2] Rui Zhang, Patrick Lochmatter, Andreas Kun and Gabor Kovacs, "Spring Roll Dielectric Elastomer Actuators for a Portable Force Feedback Glove," Proceedings of SPIE Vol. 6168, 61681T, (2006).

[3] Q. Pei, R. Pelrine, S. Stanford, R. Kornbluh, M. Rosenthal, Synthetic Metals, 135136, 129- 131(2003). 
[4] Q. Pei, R. Pelrine, S. Stanford, R. Kornbluh, M. Rosenthal, K. Meijer, R. "Full, Smart Structures and Materials," Proc. of SPIE Vol. 4698, 246 (2002).

[5] Guggi Kofod, "The static actuation of dielectric elastomer actuators: how does pre-stretch improve actuation?," J. Physics. D: Appled Physics. 41215405 (11pp) (2008)

[6] Woosang JUNG, Yutaka TOI, "Computational Modeling of Electromechanical Behaviors of Dielectric Elastomer Actuators," Proceedings of the International MultiConference of Engineers and Computer Scientists 2010 Vol III, IMECS 2010, March 17-19, 2010, Hong Kong.

[7] R. E. Pelrine, R. D. Kornbluh, J. P. Joseph, Sensors and Actuators A 64, 77 (1998).

[8] J. S. Plante, S. Dubowsky, International Journal of Solids and Structures 43, 7727 (2006).

[9] A. N. Norris, Applied Physics Letters 92, 026101 (2008).

[10] X. Zhao, Z. Suo, Applied Physics Letters 91 (2007).

[11] Mickael Moscardo, Xuanhe Zhao, Zhigang Suo,_ and Yuri Lapusta, "On designing dielectric elastomer actuators," Journal of Applied Physics 104, 093503, (2008).

[12] Sandeep Pemmaraju, " High voltage charge pump circuit for an ion mobility spectrometer", submitted for the degree of Master of science in Engineering, Electrical Engineering, Boise State University, 2004.

[13] Janusz A. Starzyk, Ying-Wei Jan, and Fengjing Qiu, "A DC-DC charge pump design based on voltage doublers" IEEE transactions on circuits and systems - I: fundamental theory and applications, vol. 48, no. 3, March 2001.

\section{APPENDIX}

function [11, 12, d1max, phic, $\mathrm{k}, \mathrm{n}]=\operatorname{actdesign}(\mathrm{p}, 11 \mathrm{relax}, 11 \max , \mathrm{r})$

$\%$ We develop this equations of state based Mat-Lab program to design spring-roll

$\%$ dielectric elastomer actuators.

$\% 11$ max is the maximum required actuator length.

$\%$ 11relax is the spring-roll dielectric elastomer actuator at relax.

$\% \mathrm{p}$ (lowr case letter)is the compressive load in Newtons.

$\% \mathrm{P}$ (upper case letter) is the dimensionless axial force.

$\%$ phic is the applied voltage at a critical state.

$\% \mathrm{~d} 1 \mathrm{max}$ is maximum actuation.

$\% \mathrm{r}$ is the radius of spring.

$\% \mathrm{n}$ number of turns of the actuator membrane around the spring.

$\% \mathrm{k}$ is the stiffness of the spring.

$\% 11,12$ and 13 are the length, width and thickness of the actuator membrane.

$\% \mathrm{mu}$ is the shear modulus of the actuator material (dielectric elastomer).

$\%$ epsilon is the dielctric of the actuator material.

$\% \mathrm{~d} 1 \mathrm{p} \& \mathrm{~d} 2 \mathrm{p}$ are the prestretches in in the length and width of the

$\%$ membrane.

$\% \mathrm{Q}$ (upper letter) is the dimensionless charge.

$\%$ Given (11max, 11relax, and p), [11, 12, d1max, phic, k]can be determined.

$\mathrm{mu}=10 .^{\wedge} 5$; 


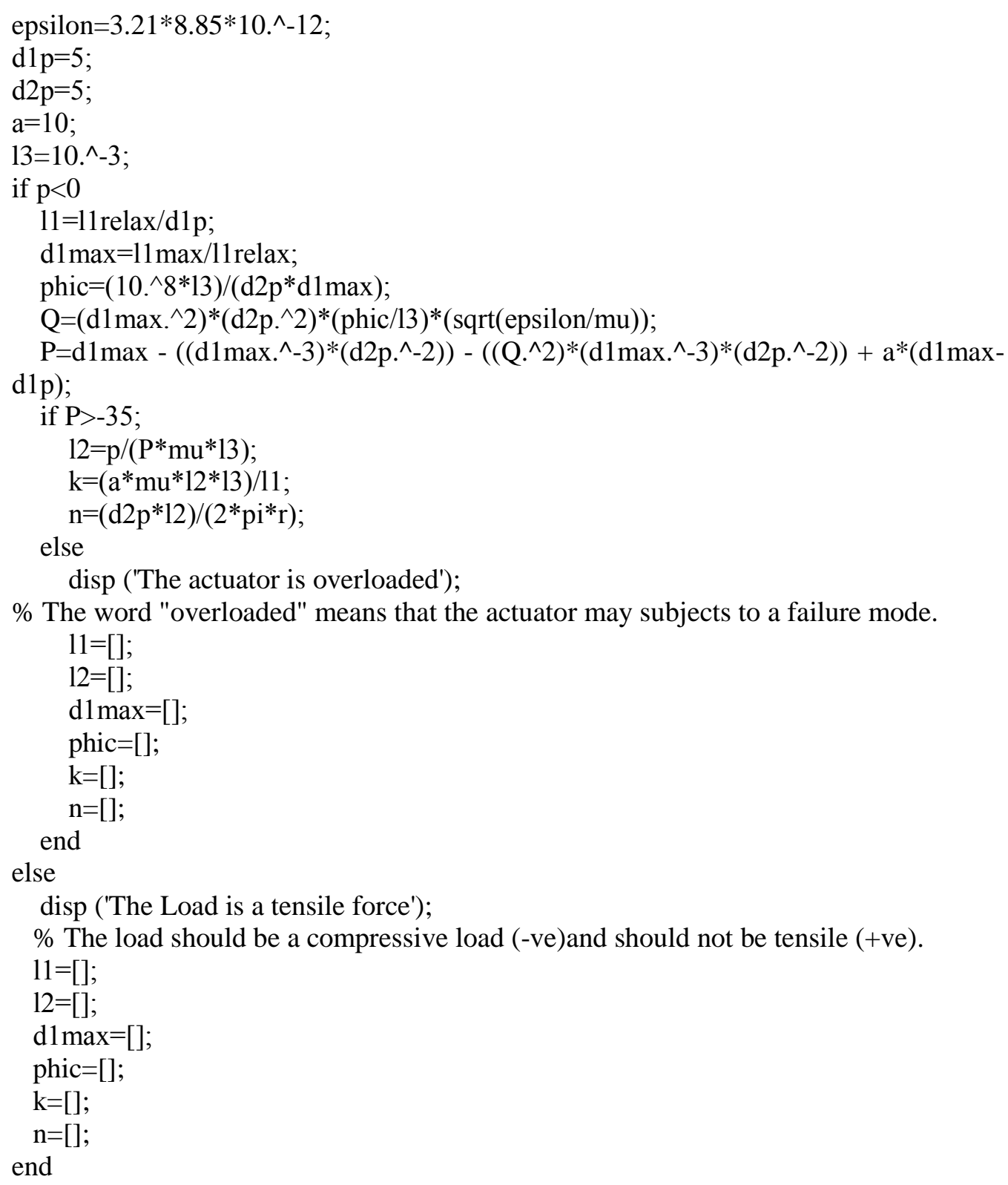

$\%$ The word "overloaded" means that the actuator may subjects to a failure mode.

$11=[]$;

$12=[]$;

$\mathrm{d} 1 \max =[]$;

phic $=[]$;

$\mathrm{k}=[]$;

$\mathrm{n}=[]$;

end

else

disp ('The Load is a tensile force');

$\%$ The load should be a compressive load (-ve)and should not be tensile (+ve).

$11=[]$;

$12=[]$;

$\mathrm{d} 1 \max =[]$;

phic=[];

$\mathrm{k}=[]$;

$\mathrm{n}=[]$

end 


\section{استحداث نظام مشغل مصنّع من مادة الإلاستومر العازل وملقوف على زنبرك

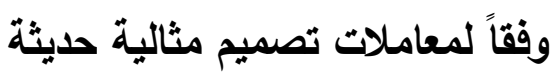

لإستحداث نظام مشغل من الالاستومر العازل الملفوف على زنبرك يجب إستحداث مشغل ذو معاملات تصميم

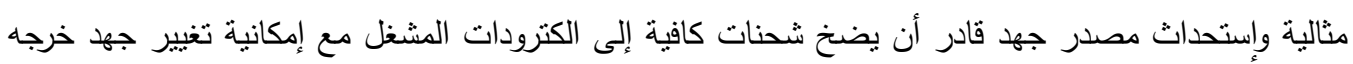

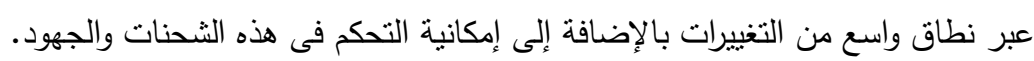

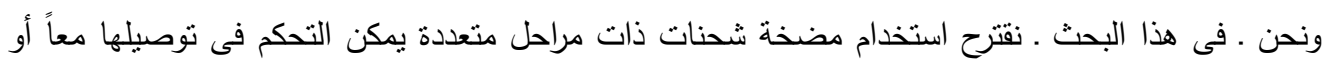

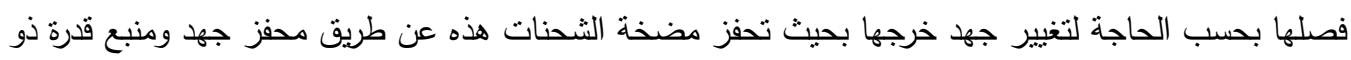

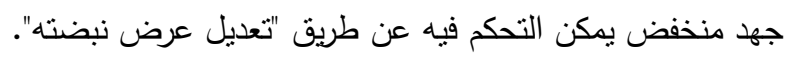

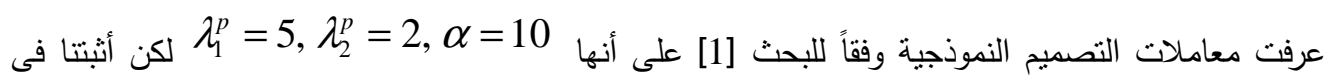
هذا البحث أن $\lambda_{2}^{p}$

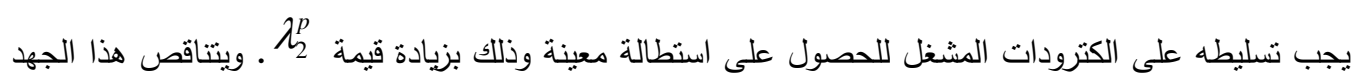

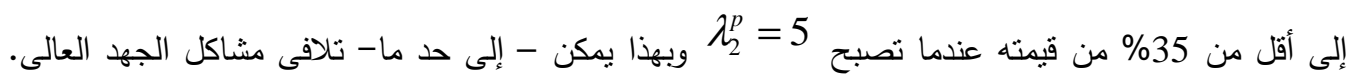

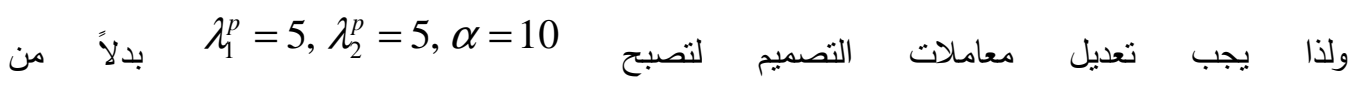
$\lambda_{1}^{p}=5, \lambda_{2}^{p}=2, \alpha=10$

لق كد أوردنا فى هذا البحث إجراءات تصميم المشغل المصنع من الإلاسنومر العازل وفقاً لمعاملات تصميم مثالية

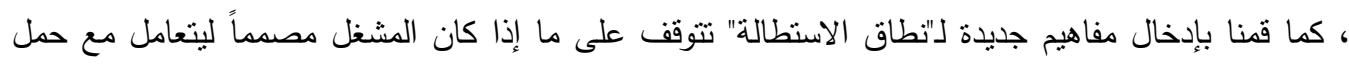

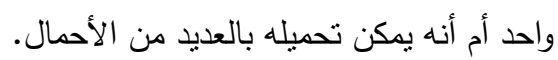

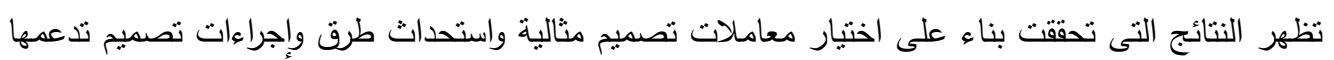

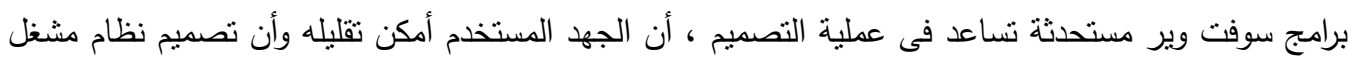
من الإلاستومر العازل الملفوف على زنبرك أصبح سهلاً وميسوراً. 